\title{
GROWTH OF HETEROTROPHE AND AUTOTROPHE POPULATIONS IN AN ISOLATED TERRESTRIAL ENVIRONMENT
}

Abstract. We consider the model, proposed by Dawidowicz and Zalasiński, describing the interactions between the heterotrophic and autotrophic organisms coexisting in a terrestrial environment with available oxygen. We modify this model by assuming intraspecific competition between heterotrophic organisms. Moreover, we introduce a diffusion of both types of organisms and oxygen. The basic properties of the extended model are examined and illustrated by numerical simulations.

1. Introduction. In this paper we consider the model proposed by Dawidowicz and Zalasiński in [D], which describes relations between heterotrophic and autotrophic organisms depending on the oxygen level in a terrestrial environment. Dawidowicz and Zalasiński in [D] assumed that production of oxygen takes place during the photosynthesis. In this model the variables $X$ and $Y$ describe the concentration of the biomass of the heterotrophic and autotrophic organisms, respectively, while $Z$ is the oxygen concentration available in the terrestrial environment. For simplicity it was also assumed that one unit of the autotrophe biomass is consumed by the use of one unit of oxygen volume. Moreover, it was assumed that there is some critical level of oxygen concentration, denoted by $K$, below which biological processes are impossible. Therefore, only an excess over the critical level takes part in the consumption process. Taking this fact into account it was assumed that the increase of the heterotrophic biomass and the decrease of the autotrophic biomass as well as the oxygen level were proportional to $X \min \left\{Y,(Z-K)^{+}\right\}$, where $(Z-K)^{+}=\max \{Z-K, 0\}$, due to consumption and respiration processes. Moreover, it was assumed that the carrying

2010 Mathematics Subject Classification: Primary 92D25; Secondary 35 B35.

Key words and phrases: autotrophes, heterotrophes, stability, diffusion. 
capacity for autotrophes was bounded due to e.g. a limited water amount. However, since in the model proposed in [D] the oxygen concentration could increase to infinity (see [FSz1] for details) we consider instead the modified model proposed by Foryś and Szymańska [FSz2], who assumed a limited amount of oxygen. To simplify the model we assume that the total concentration of the free oxygen and carbon dioxide is spatially homogeneous and denote it by $L$. Naturally, autotrophes assimilate carbon dioxide from the air and produce oxygen during the photosynthesis process. Therefore, the increase of the oxygen level is proportional to $Y(L-Z)$.

We further modify the Foryś-Szymańska model by assuming that intraspecific competition between heterotrophes is present in the ecosystem. Hence, in the proposed model, the decrease of the heterotrophic biomass is proportional to $X^{2}$. Thus, we write $-b X^{2}$ instead of $-b X$ (considered by Dawidowicz and Zalasiński as well as by Foryś and Szymańska) in (1.1) below. Moreover, we add a positive diagonal diffusion matrix. Therefore, the proposed model has the following form:

$$
\begin{aligned}
& \frac{\partial X}{\partial t}=D_{1} \Delta X+a X \min \left\{Y,(Z-K)^{+}\right\}-b X^{2}, \\
& \frac{\partial Y}{\partial t}=D_{2} \Delta Y+c Y(M-Y)-d X \min \left\{Y,(Z-K)^{+}\right\}, \\
& \frac{\partial Z}{\partial t}=D_{3} \Delta Z+e Y(L-Z)-f X \min \left\{Y,(Z-K)^{+}\right\},
\end{aligned}
$$

where $X=X(t, x)$ and $Y=Y(t, x)$ describe the concentration of the heterotrophic and autotrophic organisms at time $t$ and at the point $x \in \Omega \subset \mathbb{R}^{n}$, respectively, while $Z=Z(t, x)$ is the concentration of free oxygen available at time $t$ and at point $x$. Moreover, the constants $a, b, c, d, e, f, K, L$ and $M$ are positive. In this paper the system (1.1)-(1.3) is considered on the $1 \mathrm{D}$ open interval $\Omega=(0, l), l>0$, with no-flux (e.g. Neumann) boundary conditions. Namely, we assume

$$
\left.\frac{\partial u}{\partial x}\right|_{x=0, l}=0 \quad \text { for } u=X, Y, Z .
$$

We have chosen the 1D case only for the sake of simplicity of presenting numerical simulations. Theorem 2.1 and the model analysis given in Section 2 also hold in 2D and 3D cases. To close the system we postulate the following initial conditions:

$$
X(0, x)=X_{0}(x), \quad Y(0, x)=Y_{0}(x), \quad Z(0, x)=Z_{0}(x) .
$$

The present paper is organised as follows. Section 2 is devoted to the basic mathematical properties of the proposed model. Section 3 presents numerical simulations performed with MATLAB. The last section, Section 4, contains a discussion and conclusions. 


\section{Model analysis}

2.1. Existence and non-negativity of solutions. In the proof of the Existence Theorem 2.1 we will use the theory of invariant regions presented in [CCS] and [S]. This theory, however, requires the reaction term of the equation to be $C^{1}$. In order to have $C^{1}$ functions on the right-hand side of (2.1) $-(2.3)$ we do the following. First, we rewrite the $\min \{\cdot, \cdot\}$ and $\max \{\cdot, \cdot\}$ functions in the form

$$
\min \{x, y\}=\frac{x+y}{2}-\frac{|x-y|}{2} \text { and } \max \{x, y\}=\frac{x+y}{2}+\frac{|x-y|}{2},
$$

respectively. Secondly, we choose a small $\varepsilon>0$ and define the function

$$
m(w)= \begin{cases}|w| & \text { for }|w|>\varepsilon, \\ \frac{3}{2 \varepsilon} w^{2}-\frac{1}{2 \varepsilon^{3}} w^{4} & \text { for }|w| \leq \varepsilon .\end{cases}
$$

Now we define the functions $\min ^{*}$ and $\max ^{*}$ by inserting the function $m(\cdot)$ instead of the modulus in the formulas for min and max, respectively. Hence, we consider the system

$$
\begin{aligned}
& \frac{\partial X}{\partial t}=D_{1} \Delta X+a X \min ^{*}\left\{Y,(Z-K)^{+}\right\}-b X^{2}, \\
& \frac{\partial Y}{\partial t}=D_{2} \Delta Y+c Y(M-Y)-d X \min ^{*}\left\{Y,(Z-K)^{+}\right\}, \\
& \frac{\partial Z}{\partial t}=D_{3} \Delta Z+e Y(L-Z)-f X \min ^{*}\left\{Y,(Z-K)^{+}\right\} .
\end{aligned}
$$

Now we are able to state and prove the existence theorem.

TheOREM 2.1. Let $X_{0}(x), Y_{0}(x)$ and $Z_{0}(x)$ be bounded and uniformly continuous. Moreover, assume that the initial data are non-negative and satisfy

$$
X_{0}(x) \leq \check{X}, \quad Y_{0}(x) \leq M \quad \text { and } \quad Z_{0}(x) \leq L,
$$

where $\check{X} \geq a P / b$ and $P=\min ^{*}\{M, L-K\}$ in case $L>K$, and $\check{X}>0$ can be an arbitrary constant otherwise. Then there exists a unique global in time solution to (2.1)-(2.3) with boundary conditions (1.4) and initial conditions (1.5). Moreover, for every $t>0$ the functions $X(t, \cdot), Y(t, \cdot) Z(t, \cdot)$ are bounded and uniformly continuous.

Proof. We claim that the system (2.1)-(2.3) admits the following bounded invariant region:

$$
\Sigma=\left\{\left(u_{1}, u_{2}, u_{3}\right) \in \mathbb{R}^{3}: 0 \leq u_{1} \leq \check{X}, 0 \leq u_{2} \leq M, 0 \leq u_{3} \leq L\right\} .
$$

This implies (cf. [CCS], [S] ) the existence and uniqueness of global in time solutions to (2.1)- (2.3). Moreover, by the definition of $\Sigma$ we obtain the nonnegativity of solutions for any initial data satisfying (2.4). 
Let us define the functions $G_{i}, i \in\{1, \ldots, 6\}$, which we use to describe the boundary of $\Sigma$ :

$$
\begin{array}{ll}
G_{1}\left(u_{1}, u_{2}, u_{3}\right)=-u_{1}, & G_{2}\left(u_{1}, u_{2}, u_{3}\right)=u_{1}-\check{X}, \\
G_{3}\left(u_{1}, u_{2}, u_{3}\right)=-u_{2}, & G_{4}\left(u_{1}, u_{2}, u_{3}\right)=u_{2}-M, \\
G_{5}\left(u_{1}, u_{2}, u_{3}\right)=-u_{3}, & G_{6}\left(u_{1}, u_{2}, u_{3}\right)=u_{3}-L,
\end{array}
$$

where $\check{X}$ is as in the theorem. Then

$$
\Sigma=\bigcap_{i=1}^{6}\left\{u \in \mathbb{R}^{3}: G_{i}(u) \leq 0\right\} .
$$

The function describing the reaction term of 2.1 -2.3 can be represented as follows:

$$
\begin{aligned}
g\left(u_{1}, u_{2}, u_{3}\right)= & \left(a u_{1} \min ^{*}\left\{u_{2},\left(u_{3}-K\right)^{+}\right\}-b u_{1}^{2},\right. \\
& c u_{2}\left(M-u_{2}\right)-d u_{1} \min ^{*}\left\{u_{2},\left(u_{3}-K\right)^{+}\right\}, \\
& \left.e u_{2}\left(L-u_{3}\right)-f u_{1} \min ^{*}\left\{u_{2},\left(u_{3}-K\right)^{+}\right\}\right) .
\end{aligned}
$$

We check that

$$
\begin{array}{ll}
\left.\nabla G_{1} \cdot g\right|_{u_{1}=0}=0 & \text { in } \Sigma, \\
\left.\nabla G_{3} \cdot g\right|_{u_{2}=0}=d u_{1} \min ^{*}\left\{0,\left(u_{3}-K\right)^{+}\right\}=0 & \text { in } \Sigma, \\
\left.\nabla G_{4} \cdot g\right|_{u_{2}=M}=-d u_{1} \min ^{*}\left\{M,\left(u_{3}-K\right)^{+}\right\} \leq 0 & \text { in } \Sigma, \\
\left.\nabla G_{5} \cdot g\right|_{u_{3}=0}=-e u_{2} L \leq 0 & \text { in } \Sigma, \\
\left.\nabla G_{6} \cdot g\right|_{u_{3}=L}=-f u_{1} \min ^{*}\left\{u_{2},(L-K)^{+}\right\} \leq 0 & \text { in } \Sigma .
\end{array}
$$

Hence, $u_{1} \geq 0, u_{2} \in[0, M]$ and $u_{3} \in[0, L]$. Moreover, if $L>K$ we have

$$
\left.\nabla G_{2} \cdot g\right|_{u_{1}=\check{X}}=a \check{X} P-b \check{X}^{2} \leq 0 \quad \text { in } \Sigma,
$$

so $u_{1} \leq \check{X}$ provided that $\check{X} \geq a P / b$, where $P=\min ^{*}\{M, L-K\}$. On the other hand, for $L \leq K$ we obtain

$$
\left.\nabla G_{2} \cdot g\right|_{u_{1}=\check{X}}=-b \check{X}^{2} \leq 0 \quad \text { in } \Sigma .
$$

This implies that $u_{1} \leq \check{X}$. Clearly, we have just shown that the vector field $g$, defined in (2.6), is tangent to $\partial \Sigma$ or is directed into $\Sigma$. Therefore, by the general theory provided in [CCS] and [S] we infer that $\Sigma$ is an invariant region, and the proof is complete.

Note that for $L \leq K$ the system 2.1) -2.3 reduces to a system of three equations where the first two are decoupled and the third is coupled only 
with the second:

$$
\begin{aligned}
& \frac{\partial X}{\partial t}=D_{1} \Delta X-b X^{2}, \\
& \frac{\partial Y}{\partial t}=D_{2} \Delta Y+c Y(M-Y), \\
& \frac{\partial Z}{\partial t}=D_{3} \Delta Z+e Y(L-Z) .
\end{aligned}
$$

Clearly, by the definition of $\Sigma$, for every initial condition satisfying (2.4) we have $Z(t, x) \leq L$ for every $t>0$ and $x \in(0, l)$. Hence $\min ^{*}\left\{Y,(Z-K)^{+}\right\}=$ $\min \left\{Y,(Z-K)^{+}\right\}=0$.

2.2. Case $L \leq K$. In this section we consider the system $2.7-(2.9)$. One immediately sees that 2.7 is simply the one-dimensional heat equation. Obviously, the intersection of invariant regions is an invariant region. Hence, the invariant region of (2.7) is simply $\{0\}$. Therefore, $X(t, x) \rightarrow 0$ as $t \rightarrow \infty$. Equation (2.8) is the Fisher equation (see e.g. [BT] for details).

2.2.1. Spatially homogeneous steady states. First consider the system (2.7)-2.9 without diffusion. As mentioned before, (2.7) and (2.8) are decoupled. Moreover, the $X$ and $Y$ variables do not depend on $Z$. It is clear that 2.7) has only one stationary solution $\bar{X}=0$, which is stable. Moreover, 2.8 is simply a logistic equation (see $\mathrm{M}$ for details). Hence, it has two stationary solutions $\bar{Y}_{1}=0$ and $\bar{Y}_{2}=M$, which are unstable and stable, respectively. Computing the values of steady states for 2.9 one concludes that $(2.7)$ (2.9) have two spatially homogeneous steady states: $\left(\bar{X}_{1}, \bar{Y}_{1}, \bar{Z}_{1}\right)=(0,0, Z)$, where of course $\tilde{Z} \leq L$ and $\left(\bar{X}_{2}, \bar{Y}_{2}, \bar{Z}_{2}\right)=(0, M, L)$. Moreover, it is clear that $\left(\bar{X}_{1}, \bar{Y}_{1}, \bar{Z}_{1}\right)$ is unstable while $\left(\bar{X}_{2}, \bar{Y}_{2}, \bar{Z}_{2}\right)$ is stable.

It is known that if a stationary solution of a system without diffusion is unstable, then the introduction to the system of a non-zero diffusion matrix does not stabilize this solution (see e.g. [M]). Therefore, $\left(\bar{X}_{1}, \bar{Y}_{1}, \bar{Z}_{1}\right)$ is unstable for 2.7)-2.9 with or without diffusion.

The stationary solution $\left(\bar{X}_{2}, \bar{Y}_{2}, \bar{Z}_{2}\right)$ is stable for the model without diffusion, and it cannot be destabilized by diffusion. More precisely, consider (2.8) - 2.9 with the linearisation matrix around the point $\left(\bar{Y}_{2}, \bar{Z}_{2}\right)$ given by

$$
A_{1}=\left[\begin{array}{cc}
-c M & 0 \\
e L & -e M
\end{array}\right] \text {. }
$$

Since $A_{1}$ is a strongly stable matrix $\left({ }^{1}\right)$ the stationary solution $\left(\bar{Y}_{2}, \bar{Z}_{2}\right)$ is a stable solution of 2.8-2.9 for an arbitrary choice of diffusion constants

$\left({ }^{1}\right)$ We say that a matrix $A_{1}$ is strongly stable if $A_{1}$ as well as $A_{1}-D$ have all eigenvalues in the open left complex half-plane, where $D$ is an arbitrary positive diagonal diffusion matrix. 
(see [C] or [SMM for more details). Therefore, $\left(\bar{X}_{2}, \bar{Y}_{2}, \bar{Z}_{2}\right)$ is stable in both cases - with or without diffusion.

2.2.2. Travelling wave solutions. As shown above, 2.7 -2.9 has two spatially homogeneous steady states, one stable and the other unstable. This suggests the possibility of the existence of travelling wave solutions. After the rescaling $Y^{*}=Y / M, t^{*}=c M t$ and $x^{*}=x\left(c M / D_{2}\right)^{1 / 2}$, 2.8 becomes the classical Fisher equation (cf. [BT]) for which there exist an infinite number of travelling wave solutions. Moreover, the wavefront solutions move with speed $v \geq v_{\min }=2\left(c M D_{2}\right)^{1 / 2}$.

Since we have travelling wave solutions for 2.8, , one would expect similar behaviour for the variable $Z(t, x)$. The numerical simulations performed in the case of $L \leq K$, presented in Section 3, illustrate the propagation of the wave solution when the whole system is considered.

2.3. Case $L>K$. First, we calculate the steady states $(\bar{X}, \bar{Y}, \bar{Z})$ using the min function. Next we choose $\varepsilon>0$ small enough to ensure that $\min *\left\{\bar{Y},(\bar{Z}-K)^{+}\right\}=\min \left\{\bar{Y},(\bar{Z}-K)^{+}\right\}$.

2.3.1. Spatially homogeneous steady states. To find spatially homogeneous steady states of 2.1 -2.3 for $L>K$ we consider three cases:

(a) $Z \leq K$, which implies $\min \left\{Y,(Z-K)^{+}\right\}=0$,

(b) $Z>K$ and $\min \left\{Y,(Z-K)^{+}\right\}=Y$,

(c) $Z>K$ and $\min \left\{Y,(Z-K)^{+}\right\}=Z-K$.

In case (a) we have one spatially homogeneous steady state $\left(\bar{X}_{1}, \bar{Y}_{1}, \bar{Z}_{1}\right)=$ $(0,0, \tilde{Z})$, where $\tilde{Z} \leq K$. Using the same technique as for $L \leq K$ it can be easily shown that $\left(\bar{X}_{1}, \bar{Y}_{1}, \bar{Z}_{1}\right)=(0,0, \tilde{Z})$ is unstable for the system 2.1 2.3 with or without diffusion.

In case (b) there may exist three spatially homogeneous steady states: $\left(\bar{X}_{1}, \bar{Y}_{1}, \bar{Z}_{1}\right)=(0,0, \tilde{Z})$ for $\tilde{Z}>K,\left(\bar{X}_{2}, \bar{Y}_{2}, \bar{Z}_{2}\right)=(0, M, L)$ and the last one, with positive coordinates

$$
\left(\bar{X}_{3}, \bar{Y}_{3}, \bar{Z}_{3}\right)=\left(\frac{a c M}{a d+b c}, \frac{b c M}{a d+b c}, L-\frac{a c f M}{e(a d+b c)}\right)
$$

provided that $M \leq L-K$ and $K<L-\frac{c M(a f+b e)}{e(a d+b c)}$, respectively. As in case (a), $\left(\bar{X}_{1}, \bar{Y}_{1}, \bar{Z}_{1}\right)$ is unstable for 2.1)-2.3 with or without diffusion. Linearising 2.1 2.3 without diffusion around $\left(\bar{X}_{2}, \bar{Y}_{2}, \bar{Z}_{2}\right)$ and calculating the roots of the characteristic polynomial one observes that there exists a positive root $\lambda=a M$. Hence, the solution $\left(\bar{X}_{2}, \bar{Y}_{2}, \bar{Z}_{2}\right)$ is unstable for the system with or without diffusion. To examine the stability of the third steady state we first consider the system (2.1)-2.3) without diffusion. Linearising (2.1)-2.3 around the stationary solution $\left(\bar{X}_{3}, \bar{Y}_{3}, \bar{Z}_{3}\right)$ we obtain the 
following linearisation matrix:

$$
A_{2}=A_{2}\left(\bar{X}_{3}, \bar{Y}_{3}, \bar{Z}_{3}\right)=\left[\begin{array}{ccc}
\frac{-a b c M}{a d+b c} & \frac{a^{2} c M}{a d+b c} & 0 \\
\frac{-b c d M}{a d+b c} & \frac{-b c^{2} M}{a d+b c} & 0 \\
\frac{-b c f M}{a d+b c} & 0 & \frac{-b c e M}{a d+b c}
\end{array}\right] .
$$

The characteristic polynomial $P(\lambda)=\operatorname{det}\left(A_{2}-\lambda I\right)$ has the form

$$
P(\lambda)=-\left(\lambda+\frac{b c e M}{a d+b c}\right) \operatorname{det}\left(A_{2}^{\prime}-I \lambda\right),
$$

where

$$
A_{2}^{\prime}=\left[\begin{array}{ll}
\frac{-a b c M}{a d+b c} & \frac{a^{2} c M}{a d+b c} \\
\frac{-b c d M}{a d+b c} & \frac{-b c^{2} M}{a d+b c}
\end{array}\right] .
$$

Clearly, $\operatorname{tr} A_{2}^{\prime}<0$ and $\operatorname{det}\left(A_{2}^{\prime}\right)>0$, thus both eigenvalues of $A_{2}^{\prime}$ have negative real parts and the steady state $\left(\bar{X}_{3}, \bar{Y}_{3}, \bar{Z}_{3}\right)$ is locally asymptotically stable.

The question arises whether this steady state is also stable for the system with diffusion or if Turing instability occurs. To answer this question we use Theorem 3.2 of $\mathrm{WL}$. We have to check the so-called minors condition, which postulates that $(-1)^{k} \operatorname{det}\left(P_{J}(C)\right) \geq 0$ for all $J \in I_{k}$ and all $k=1, \ldots, n$, where $I_{k}=\left\{1 \leq i_{1}<\cdots<i_{k} \leq n\right\}$ and $n$ is the size of the linearisation matrix $C$ (in our case $n=3$ ). Here $P_{J}(C)$, where $J=\left\{i_{1}, \ldots, i_{k}\right\}$, denotes the $k \times k$ principal submatrix of $C$,

$$
P_{J}(C)=\left[\begin{array}{cccc}
c_{i_{1} i_{1}} & c_{i_{1} i_{2}} & \cdots & c_{i_{1} i_{k}} \\
c_{i_{2} i_{1}} & c_{i_{2} i_{2}} & \cdots & c_{i_{2} i_{k}} \\
\vdots & \vdots & \ddots & \vdots \\
c_{i_{k} i_{1}} & c_{i_{k} i_{2}} & \cdots & c_{i_{k} i_{k}}
\end{array}\right] .
$$

It is clear that in the present case all principal minors of rank 1 are negative so $(-1) \operatorname{det}\left(P_{J}\left(A_{2}\right)\right)>0$ for $J=\{1\},\{2\}$ and $\{3\}$. For $k=2$ we check three minors:

- for $J=\{1,2\}$ we have

$$
B:=(-1)^{2} \operatorname{det}\left(P_{J}\left(A_{2}\right)\right)=a b c^{2} M^{2} /(a d+b c)>0,
$$

- for $J=\{1,3\}$ we have

$$
(-1)^{2} \operatorname{det}\left(P_{J}\left(A_{2}\right)\right)=a b^{2} c^{2} e M^{2} /(a d+b c)^{2}>0,
$$

- for $J=\{2,3\}$ we have

$$
(-1)^{2} \operatorname{det}\left(P_{J}\left(A_{2}\right)\right)=b^{2} c^{3} e M^{2} /(a d+b c)^{2}>0 .
$$


For $k=3$ we calculate $(-1)^{3} \operatorname{det}\left(A_{2}\right)=b c e M B /(a d+b c)>0$. This shows that the minors condition is satisfied. Since $A_{2}$ is stable, we infer from Theorem 3.2 of [WL] that $A-D$ is stable for all diagonal diffusion matrices $D>0$. Therefore, the steady state $\left(\bar{X}_{3}, \bar{Y}_{3}, \bar{Z}_{3}\right)$ is stable for every set of diffusion coefficients, and Turing instability cannot occur.

In the last case (c), when all three equations are coupled, we have one spatially homogeneous steady state $\left(\bar{X}_{2}, \bar{Y}_{2}, \bar{Z}_{2}\right)=(0, M, L)$, as for case (b). However, now $M>L-K$. Using the same techniques as in case (b) it is easy to show that $\left(\bar{X}_{2}, \bar{Y}_{2}, \bar{Z}_{2}\right)$ is unstable for (2.1)-(2.3) with or without diffusion. Moreover, depending on parameter values, there may exist one or two more positive homogeneous steady states

$$
\left(\bar{X}_{4,5}, \bar{Y}_{4,5}, \bar{Z}_{4,5}\right)=\left(\frac{a}{b}(\widehat{Z}-K), M-\frac{d e}{c f}(L-\widehat{Z}), \widehat{Z}\right),
$$

where $\widehat{Z}$ is one of the roots of the polynomial

$$
\begin{aligned}
-\left(\frac{d e^{2}}{c f}+\frac{a f}{b}\right) Z^{2}+\left(2 L \frac{d e^{2}}{c f}+2 K\right. & \left.\frac{a f}{b}-e M\right) Z \\
& +e M L-\frac{d e^{2}}{c f} L^{2}-\frac{a f}{b} K^{2}=0 .
\end{aligned}
$$

In order to ensure that 2.10$)$ is/are the steady state(s) of $(2.1)-(2.3)$ the $\operatorname{root}(\mathrm{s})$ of the polynomial (2.11) has/have to meet the following conditions:

$$
\widehat{Z}>K, \quad \min \{Y, \widehat{Z}-K\}=\widehat{Z}-K \quad \text { and } \quad \widehat{Z}<L .
$$

Of course, depending on the coefficients, there also might exist some nonhomogeneous steady states for 2.1) $-(2.3)$.

3. Numerical simulations. In this section, we present some numerical simulations for the model (2.1)-2.3) in a 1D space domain. The computations were performed with MATLAB programming language using the parameter set-up given in Table 1. In all simulations we implemented the Neumann boundary conditions. The size of the domain $\Omega$ and the time interval were chosen separately for each figure to better illustrate the results.

Table 1. Simulation parameters

\begin{tabular}{|c|c|c|c|c|c|c|c|c|c|c|c|c|}
\hline & \multicolumn{10}{|c|}{ Parameters } \\
\cline { 2 - 16 } Set No. & $a$ & $b$ & $c$ & $d$ & $e$ & $f$ & $K$ & $L$ & $M$ & $D_{1}$ & $D_{2}$ & $D_{3}$ \\
\hline I & 7 & 5 & 0.5 & 3 & 10 & 5 & 20 & 10 & 20 & 0.1 & 0.01 & 10 \\
\hline II & 1 & 1 & 2.1 & 7.4 & 4.4 & 9.2 & 7.4 & 8 & 1 & 1.82 & 0.102 & 28.4 \\
\hline III & 1 & 1 & 0.1 & 0.1 & 0.1 & 0.1 & 7 & 7.9 & 1 & 0.1 & 0.01 & 10 \\
\hline IV & 1 & 1 & 2 & 4 & 3 & 2 & 5 & 20 & 1 & 0.1 & 0.01 & 10 \\
\hline
\end{tabular}




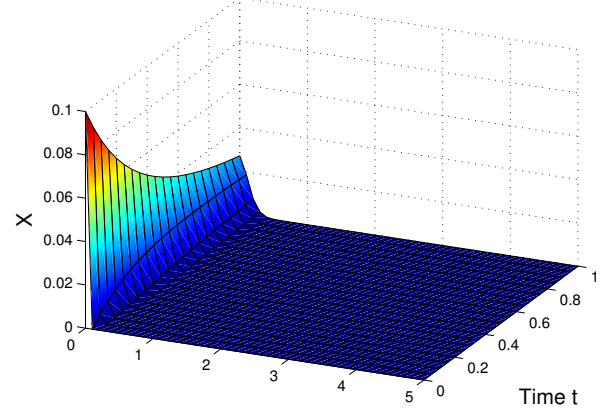

Distance $x$
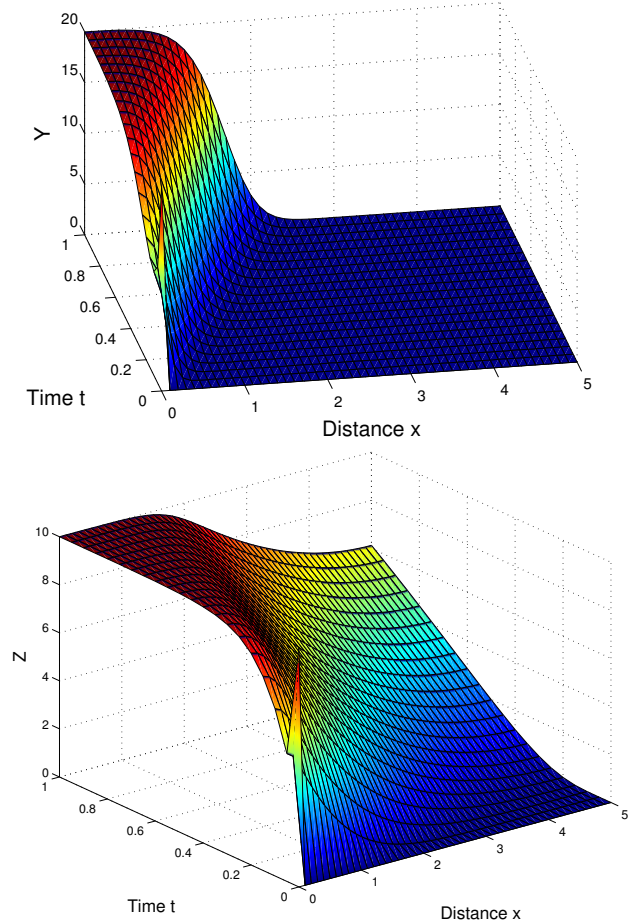

Fig. 1. Results of simulations for parameter set I. The initial condition was described in the text.

First, let us consider parameter set I in Table 1. Clearly, $L \leq K$, hence, as shown analytically in Section 2.2.1, there exists a half-line of unstable stationary solutions $\left(\bar{X}_{1}, \bar{Y}_{1}, \bar{Z}_{1}\right)=(0,0, \tilde{Z})$, where $\tilde{Z} \leq L$ (cf. case (a) in Section 2.3.1) and one stable stationary solution $\left(\bar{X}_{2}, \bar{Y}_{2}, \bar{Z}_{2}\right)=(0, M, L)$. Moreover, 2.8) has a travelling wave solution for an appropriate initial condition. To illustrate this phenomenon we take as the initial condition a profile such that $X(0, x)=0.1, Y(0, x)=M, Z(0, x)=L$ for $x<0.1$ and $X(0, x)=Y(0, x)=Z(0, x)=0$ otherwise. Additionally, to better present 
the propagation of the initial profile we take $\bar{\Omega}=[0,5]$. As suggested in Section 2.2.2 in Fig. 1 we see that the travelling wave solution propagates not only for equation (2.8) (with wave speed $v \geq 2\left(c M D_{2}\right)^{1 / 2}$ ) but also for (2.9) and of course it does not propagate for (2.7).

In the case when $L>K$ (Section 2.3) we considered three subcases. It is clear that if $Z \leq K$ we only have a half-line of unstable stationary solutions $\left(\bar{X}_{1}, \bar{Y}_{1}, \bar{Z}_{1}\right)=(0,0, \tilde{Z})$, where $\tilde{Z} \leq K<L$. Clearly, for $Y<Z-K$ there exists the half-line of unstable stationary solutions $\left(\bar{X}_{1}, \bar{Y}_{1}, \bar{Z}_{1}\right)=(0,0, \tilde{Z})$, the unstable stationary solution $(0, M, L)$ and the stable stationary solution $\left(\bar{X}_{3}, \bar{Y}_{3}, \bar{Z}_{3}\right)$. For $Y>Z-K$ we still have the unstable stationary solutions $\left(\bar{X}_{1}, \bar{Y}_{1}, \bar{Z}_{1}\right)=(0,0, \tilde{Z})$, the unstable stationary solution $(0, M, L)$ but we can also have additional stationary points $\left(\bar{X}_{4,5}, \bar{Y}_{4,5}, \bar{Z}_{4,5}\right)$. It should be noted here that the existence and stability of $\left(\bar{X}_{4,5}, \bar{Y}_{4,5}, \bar{Z}_{4,5}\right)$ strongly depends on model parameters. Since the analytical analysis of the existence and stability of 2.10 is complex, we investigated it numerically. First, to decrease the number of parameters and speed up the computation process we rescale

$$
X^{*}=\tilde{a} X, \quad Y^{*}=\frac{Y}{M}, \quad Z^{*}=\frac{Z}{M} \quad \text { and } \quad t^{*}=\tilde{b} t .
$$

Next, setting

$$
\tilde{a}=\frac{a M}{b}, \quad \tilde{b}=\frac{1}{a M}, \quad K^{*}=\frac{K}{M} \quad \text { and } \quad L^{*}=\frac{L}{M}
$$

we obtain the following system of equations:

$$
\begin{aligned}
& \frac{\partial X^{*}}{\partial t^{*}}=D_{1}^{*} \Delta X^{*}+X^{*} \min ^{*}\left\{Y^{*},\left(Z^{*}-K^{*}\right)^{+}\right\}-X^{2}, \\
& \frac{\partial Y^{*}}{\partial t^{*}}=D_{2}^{*} \Delta Y^{*}+\frac{c}{a} Y^{*}\left(1-Y^{*}\right)-\frac{d}{b} X^{*} \min ^{*}\left\{Y^{*},\left(Z^{*}-K^{*}\right)^{+}\right\}, \\
& \frac{\partial Z^{*}}{\partial t^{*}}=D_{3}^{*} \Delta Z^{*}+\frac{e}{a} Y^{*}\left(L^{*}-Z^{*}\right)-\frac{f}{b} X^{*} \min ^{*}\left\{Y^{*},\left(Z^{*}-K^{*}\right)^{+}\right\},
\end{aligned}
$$

where $D_{i}^{*}=\frac{D_{i}}{a M}, i \in\{1,2,3\}$. Setting

$$
c^{*}=\frac{c}{a}, \quad d^{*}=\frac{d}{b}, \quad e^{*}=\frac{e}{a}, f^{*}=\frac{f}{b}
$$

and dropping the superscript * (cf. beginning of Section 2.3), in the above equations we obtain the system (2.1) - 2.3) with $a=b=M=1$.

We investigated the following range of parameters: $c, d, e, f, K, L \in[0,10]$. These parameters were chosen randomly from a uniform continuous distribution on $[0,10]$ (below we write $U(a, b)$ instead of uniform continuous distribution on $[a, b]$ for short), with step 0.1. For selected parameters we first check whether the polynomial defined by 2.11) has at least one positive root $\widehat{Z}$. Then we calculate $\widehat{X}$ and $\widehat{Y}$ and examine condition (2.12). If it is satisfied we compute the eigenvalues of the linearisation matrix (denoted by $S$ ). If 
all eigenvalues have negative real parts we inspect if $S$ is strongly stable by checking the minors condition. If $S$ is not strongly stable we look for a set of diffusion coefficients $\left(D_{1}, D_{2}, D_{3}\right)$ such that the matrix $S-\operatorname{diag}\left(D_{1}, D_{2}, D_{3}\right)$ is unstable, i.e. has at least one eigenvalue with positive real part. The parameter $D_{1}$ is chosen from $U(0,10)$ with step 0.01 , while $D_{2}$ and $D_{3}$ from $U(0,1)$ with step 0.001 and $U(0,1000)$ with step 0.1 , respectively. We chose such distributions to emphasize the fact that the diffusion of oxygen is the fastest process we consider, moreover we assumed that heterotrophes get around faster than autotrophes. As a result of the above procedure we obtain a large number of parameter sets for which Turing instability exists. In this paper we consider only one set of reaction term parameters since a similar qualitative behaviour of the system was observed for other sets of parameters. To better illustrate the numerical simulation results we chose $\bar{\Omega}=[0,10]$.

In Fig. 2 the numerical simulations performed for parameter set II are presented. Clearly, as expected, after some time solutions $X, Y$ and $Z$ tend to the spatially inhomogeneous solution and pattern formation can be observed. To better understand the behaviour of the system we first fix all parameters except for $D_{1}$. It appears that for $D_{1}$ chosen from the interval $(0,10)$ with step 0.01 we always observe Turing instability. Nevertheless, we have not observed any large changes in the system's behaviour. When we fix all parameters as in II except for $D_{2}$, as in the previous case, for $D_{2} \in[0.001,0.179]$ (chosen with step 0.001) we observe pattern formation, but we do not observe it for $D_{2} \in[0.180,1]$ chosen with the same step. This time the value of $D_{2}$ influences the number of the observed humps in the pattern. Generally, lower $D_{2}$ values give a denser pattern (compare Figs. 2 and 3 ). In the case of a free parameter $D_{3}$ chosen from $[0.1,1000]$ with step 0.1 once again we always observe inhomogeneous stable solutions and again the changes in the $D_{3}$ coefficient bring about changes in the density of the pattern. As for the previous case, lower values of $D_{3}$ trigger a more dense pattern: compare Figs. 2 and 4 .

In Fig. 5 we illustrate the fact that the solution $\left(\bar{X}_{4}, \bar{Y}_{4}, \bar{Z}_{4}\right)$ can be stable under some conditions. More precisely, for parameter set III there exists only one positive root of polynomial (2.11). Moreover, the linearisation matrix at this steady state is strongly stable, so Turing instability cannot occur.

To illustrate the wave propagation phenomena we choose parameter set IV. For this set one observes travelling wave solutions with respect to all variables. Here we take the spatial domain $\bar{\Omega}=[0,5]$ and the initial profile such that $X(0, x)=0.3333, Y(0, x)=0.3333, Z(0, x)=19.7778$ for $x<0.1$, and $X(0, x)=Y(0, x)=Z(0, x)=0$ otherwise. Indeed, in Fig. 6 the propagation of the initial profile is shown. 

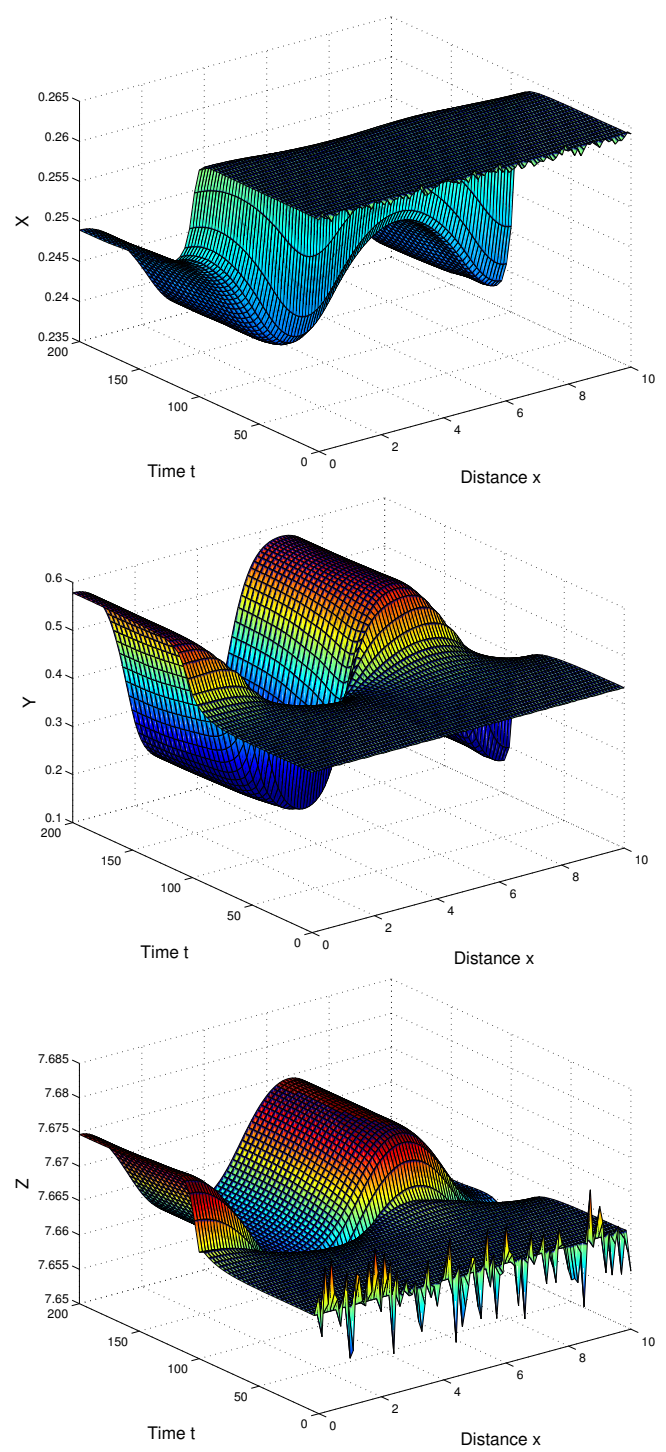

Fig. 2. Results of simulations for parameter set II. The initial condition: a perturbation of $\left(\bar{X}_{4}, \bar{Y}_{4}, \bar{Z}_{4}\right)$.

4. Conclusions. In this paper we have presented a modification of the model presented earlier in the literature, $[\mathrm{D}]$. More precisely, first, we follow the modification introduced by Foryś and Szymańska in [FSz1] and [FSz2] and put in (1.3) the term $e Y(L-Z)$ instead of $e Y(M-Y)$; secondly, we replace the term $-b X$ in 1.1 by $-b X^{2}$ to reflect intraspecific competition within the heterotrophe population. Finally we add a positive diffusion matrix to emphasize the fact that the organisms can move and oxygen diffuses. 

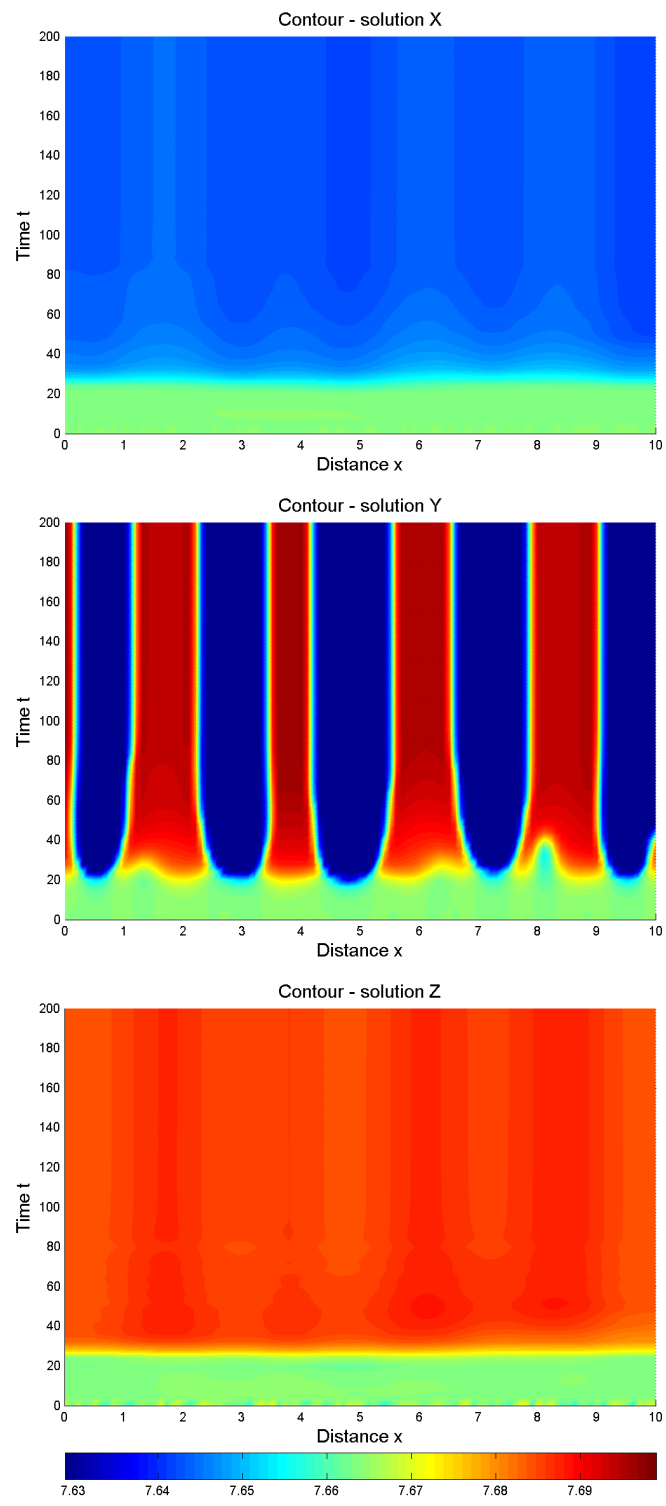

Fig. 3. Results of simulations for modified $\left(D_{2}=0.001\right)$ parameter set II. The initial condition: a perturbed value of $\left(\bar{X}_{4}, \bar{Y}_{4}, \bar{Z}_{4}\right)$. The colour in the middle of the colourbar corresponds to the value of steady state.

We have proved that the solutions of the new model $11.1-(1.3)$ exist globally, are non-negative and uniformly bounded in time. Moreover, we have found that depending on the model parameters there exist two, three, four or even five homogeneous steady states. Of course their stability strongly depends on the model parameters. 

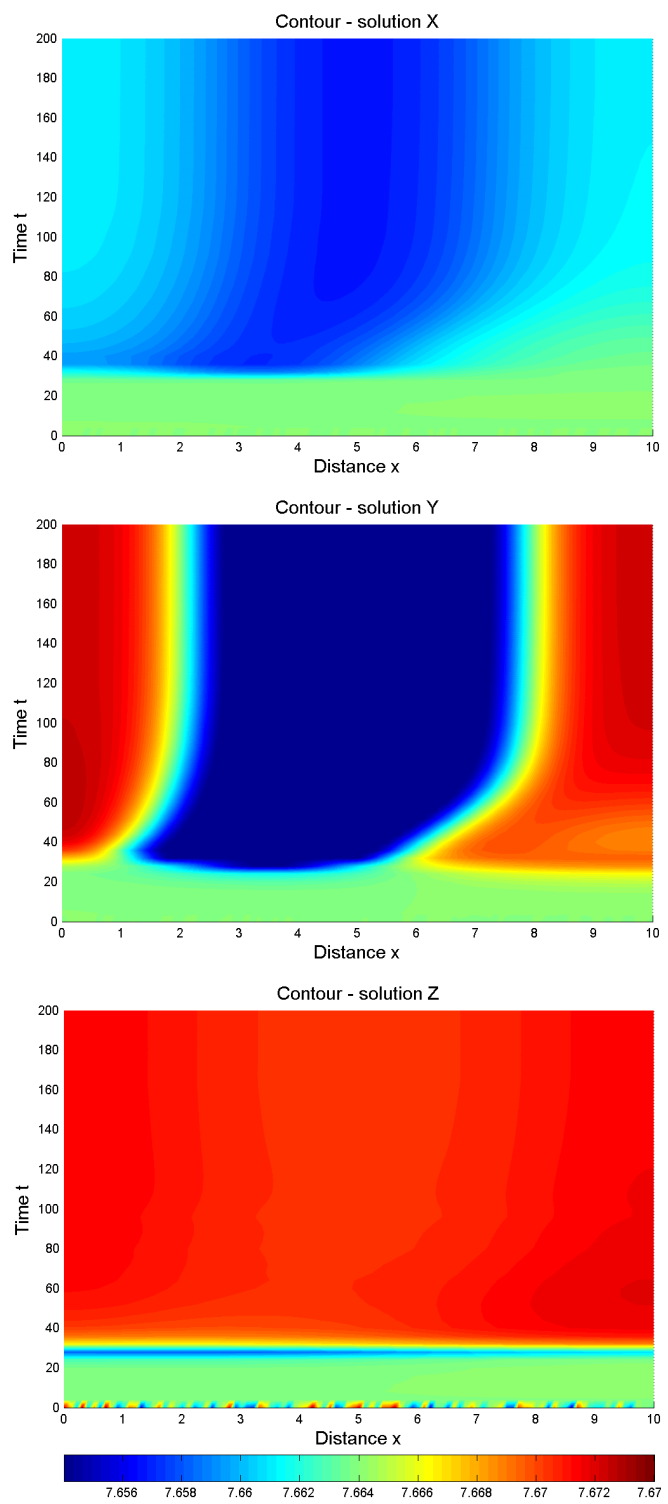

Fig. 4. Results of simulations for modified $\left(D_{3}=1000\right)$ parameter set II. The initial condition: a perturbed value of $\left(\bar{X}_{4}, \bar{Y}_{4}, \bar{Z}_{4}\right)$. The colour in the middle of the colourbar corresponds to the value of steady state.

From the biological point of view this means that if, for example, we want to colonize a low oxygen environment by introducing new populations (both autotrophe and heterotrophe), or if we try to regulate the interactions between coexisting organisms, we obtain different effects, depending on the way we do that. More precisely, if we modify the critical level of oxygen 

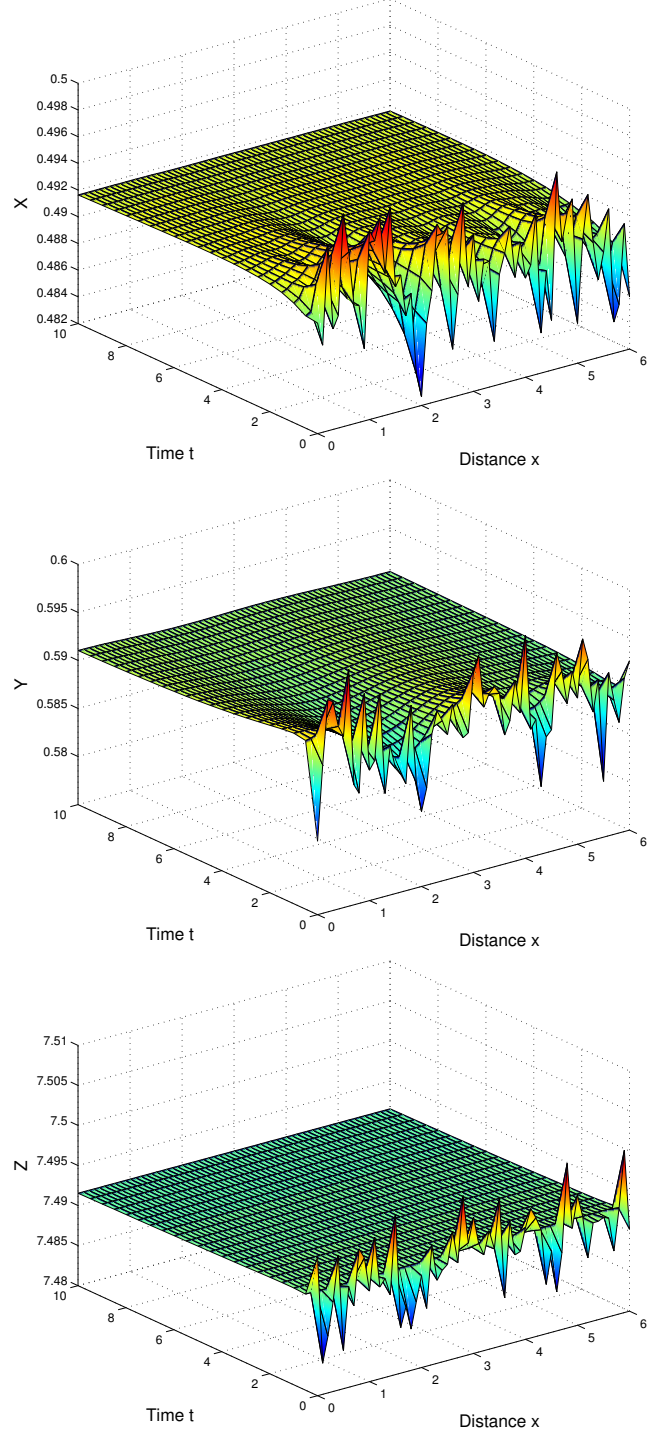

Fig. 5. Result of simulation for parameter set III. The initial condition: a perturbed value of $\left(\bar{X}_{4}, \bar{Y}_{4}, \bar{Z}_{4}\right)$.

concentration required by the heterotrophes for the respiration process $(K)$ by, say, a genetic modification, we actually change the long time behaviour of the whole system. Clearly, we have proved in Section 2 that for $L \leq K$ the heterotrophe population dies out independently of the other model parameters, while the concentrations of autotrophes and of oxygen normalize in time at the levels $M$ and $L$, respectively. Moreover, as shown in Fig. 1 , the whole accessible space is colonized by autotrophes. On the other hand, 

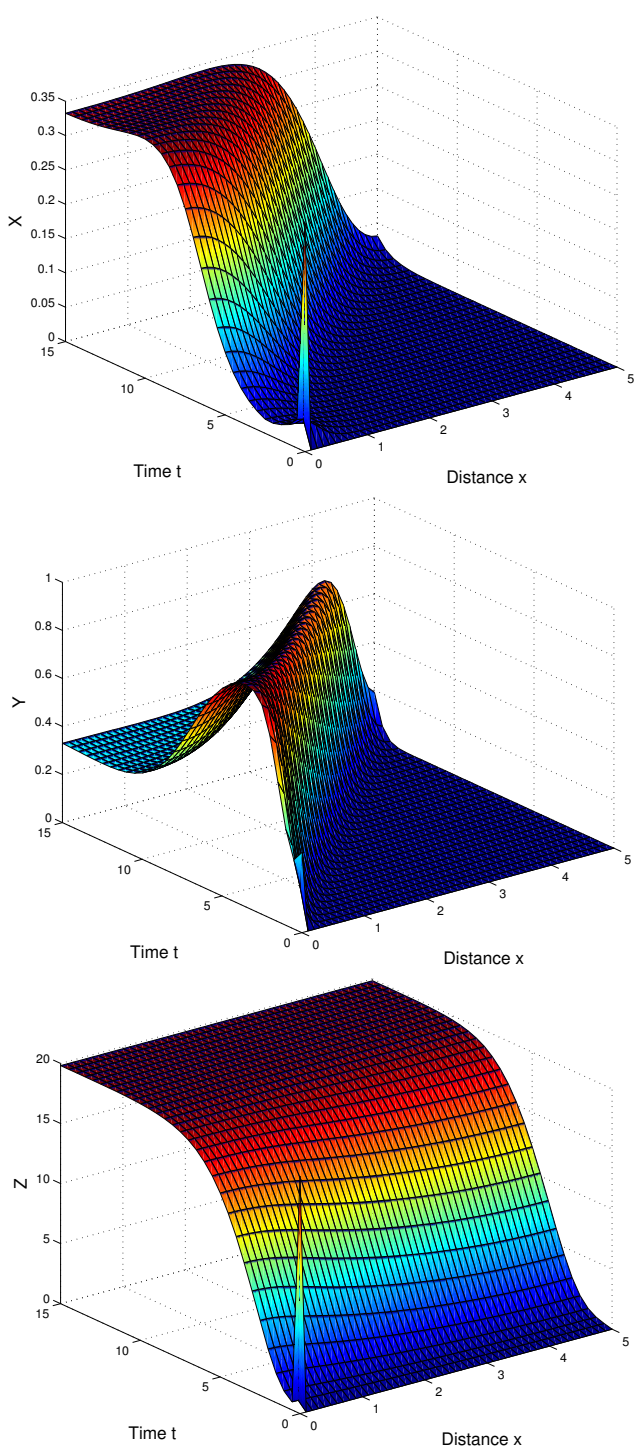

Fig. 6. Results of simulations for parameter set IV. The initial condition: the profile described in the text.

the results of Sections 2 and 3 indicate that if we are able to decrease $K$, by genetic modification of heterotrophes or by introducing to the system species with lower demand for oxygen, then under some conditions we observe two different behaviours. More precisely, the environment in question can be fully colonized by coexisting autotrophe and heterotrophe populations (see Figs. 56 6), or regions of different concentrations of entities appear as illustrated in Figs. 24. 
Summarizing, we have observed a number of behaviours of solutions for equations (1.1)-(1.3), which implies that the proposed model is quite general and can describe various phenomena:

1. The possibility of development of life in an uncolonized environment since the trivial solution $(0,0,0)$ is unstable for an arbitrary set of parameters.

2. The extinction of heterotrophes and survival of autotrophes if the maximal amount of oxygen in the environment is smaller than its quantity needed by heterotrophes to survive, i.e. $L<K$, similarly to the models proposed by Dawidowicz and Zalasiński in [D] and Foryś and Szymańska in [FSz1] and [FSz2].

3. Travelling wave solutions describing the settlement by new species of the uncolonized environment.

4. Turing instability and the creation of spatial patterns and the coexistence of both populations considered.

The approach presented above offers exciting possibilities for future work. One can generalize this model by assuming that the total concentration of oxygen and carbon dioxide is not constant. This assumption e.g. allows us to introduce environmental pollution to the model. Moreover, the term $-g(x, t) Z$ in 1.3 can be added to model the phenomena such as fire.

Acknowledgements. This article is an extended version of a work presented by the authors at the 15th National Conference on Application of Mathematics in Biology and Medicine. M. J. Piotrowska's research was supported by a Marie Curie European Reintegration Grant within the 7th European Community Framework Programme, grant agreement No. PERG03GA-2008-230993, by the Polish Ministry of Science and Higher Education, grant No. N N201 362536 and by the Foundation for Polish Science within Start Programme, while P. P. Szopa's work was supported by the Polish Ministry of Science and Higher Education, grant No. N N201 547638.

\section{References}

[BT] P. K. Brazhnik and J. J. Tyson, On travelling wave solutions of Fisher's equation in two spatial dimensions, SIAM J. Appl. Math. 60 (1999), 371-391.

[CCS] K. Chueh, C. Conley C. and J. Smoller, Positively invariant regions for systems of nonlinear diffusion equations, Indiana Univ. Math. J. 26 (1977), 373-392.

[C] G. W. Cross, Three types of matrix stability, Linear Algebra Appl. 20 (1978), 253-263.

[D] A. L. Dawidowicz and J. L. Zalasiński, A model of participation of autotropic and heterotropic organisms in phenomenon of life, in: Proceedings of the Twelfth National Conference on Application of Mathematics in Biology and Medicine 
(Koninki, 2006), University of Computer Engineering and Telecommunication in Kielce, 2006, 37-39.

[FSz1] U. Foryś and Z. Szymańska, Analysis of heterotrophic-autotrophic organisms model, in: Proceedings of the Thirteenth National Conference on Application of Mathematics in Biology and Medicine (Serpelice nad Bugiem, 2007), Jagiellonian University, Kraków, 2007, 43-48.

[FSz2] U. Foryś and Z. Szymańska, Models of interactions between heterotrophic and autotrophic organisms, Appl. Math. (Warsaw) 36 (2009), 279-294.

[M] J. D. Murray, Mathematical Biology II: Spatial Models and Biomedical Applications, 3rd ed., Springer, New York, 2001.

[SMM] R. A. Satnoianu, M. Menzinger and P. K. Maini, Turing instabilities in general systems, J. Math. Biol. 41 (2000), 493-512.

[S] J. Smoller, Shock Waves and Reaction-Diffusion Equations, Springer, New York, 1983.

[WL] L. Wang and M. Y. Li, Diffusion driven instability in reaction-diffusion systems, J. Math. Anal. Appl. 254 (2001), 138-153.

Piotr Paweł Szopa, Monika Joanna Piotrowska

Institute of Applied Mathematics and Mechanics

Faculty of Mathematics, Informatics and Mechanics

University of Warsaw

Banacha 2

02-097 Warszawa, Poland

E-mail: szopa@mimuw.edu.pl

monika@mimuw.edu.pl

Received on 17.5.2010;

revised version on 22.11.2010 